\title{
Daily snacking frequency is associated with age, working pattern and dietary nutritional profile of professional workers aged between 30 and 65 years old
}

\author{
Y. Jin ${ }^{1}$ and T. Birchall ${ }^{1}$ \\ ${ }^{1}$ School of Health Sciences, Liverpool Hope University, Liverpool. L16 9JD.
}

There is an emerging body of studies showing the association of snacking behaviour with weight status or nutritional status in general populations ${ }^{(1,2)}$. However, there is a lacking of evidence examining the link of snacking frequency with nutritional status in urbanliving professional workers. This study investigated the association of daily snacking frequency with nutritional status amongst professional workers residing in the North West of England.

35 professional workers (M 13, F 22) aged 30-65 years old were recruited via random selection from Liverpool and its surrounding areas. Laboratory and anthropometric measures of CVD risk were assessed including blood pressure, measures of central obesity and biomarkers of fasting capillary blood glucose and lipid profile. Dietary intake profiles including total energy, nutrients and snacking behaviours were recorded using a validated 3-day diet diary and analysed using a dietary assessment software Microdiet. Information on working hours per week was collected using a sociodemographic questionnaire. Variation in nutritional status amongst all snacking-frequency groups was assessed by Univariate analysis with Bonferroni post hoc test using SPSS (v 22). The statistical significance was set at $0 \cdot 05$.

The snack items consumed comprised hot and cold beverages, wine and beer, savoury snacks, fruit and vegetables, toast, sandwich, crumpet, humus, bread sticks, cereals, cereal bars, dairy foods, sweets, confectioneries, ice cream, nuts, raisins, fruit jelly, yogurt. Significant variations existed in age, working hours/week and dietary nutritional profiles across all snack-frequency groups. Only statistically significant data are presented in the Table below.

\begin{tabular}{|c|c|c|c|c|c|c|c|c|}
\hline \multirow[b]{3}{*}{ Parameters } & \multicolumn{8}{|c|}{ Snacking Frequency/day* } \\
\hline & \multicolumn{2}{|l|}{ Once } & \multicolumn{2}{|l|}{ Twice } & \multicolumn{2}{|c|}{ Three times } & \multicolumn{2}{|c|}{ Four times } \\
\hline & Mean & SD & Mean & SD & Mean & SD & Mean & SD \\
\hline Total Energy intake (Kcal/day) & $1575 \cdot 56^{\mathrm{a}}$ & $114 \cdot 04$ & $1955 \cdot 07^{\mathrm{ab}}$ & $557 \cdot 82$ & $2131.47^{\mathrm{ab}}$ & $506 \cdot 81$ & $2413 \cdot 92^{\mathrm{b}}$ & $802 \cdot 88$ \\
\hline Dietary total sugar intake (g/day) & $54.97^{\mathrm{a}}$ & 15.97 & $80 \cdot 29^{\mathrm{ab}}$ & 31.08 & $103 \cdot 58^{\mathrm{b}}$ & $42 \cdot 39$ & $108 \cdot 83^{\mathrm{b}}$ & $44 \cdot 84$ \\
\hline Dietary protein intake (g/day) & $56 \cdot 23^{\mathrm{a}}$ & 1.48 & $72 \cdot 88^{\mathrm{ab}}$ & 1.33 & $72 \cdot 51^{\mathrm{ab}}$ & 1.32 & $104 \cdot 71^{b}$ & 1.82 \\
\hline Dietary total cholesterol (g/day) & $95 \cdot 02^{\mathrm{a}}$ & $3 \cdot 29$ & $153 \cdot 39^{\mathrm{ab}}$ & 1.83 & $172 \cdot 07^{\mathrm{ab}}$ & $2 \cdot 07$ & $312 \cdot 10^{\mathrm{b}}$ & 1.60 \\
\hline Dietary Retinol Equivalent ( $\mu \mathrm{g} /$ day) & $661 \cdot 07^{\mathrm{ab}}$ & 251.71 & $553 \cdot 37^{\mathrm{a}}$ & $337 \cdot 31$ & $840 \cdot 48^{\mathrm{b}}$ & 399.98 & $26 \cdot 97^{\mathrm{b}}$ & 299.90 \\
\hline Dietary Total carotene $(\mu \mathrm{g} /$ day $)$ & $2238 \cdot 72^{\mathrm{b}}$ & $2 \cdot 19$ & $1288 \cdot 25^{\mathrm{ab}}$ & 2.59 & $2691 \cdot 53^{\mathrm{b}}$ & $2 \cdot 51$ & $436 \cdot 52^{\mathrm{a}}$ & 4.68 \\
\hline Dietary Vitamin $B_{2}$ ( $\mu \mathrm{g} /$ day) & $1.66^{\mathrm{ab}}$ & $0 \cdot 84$ & $1 \cdot 16^{\mathrm{a}}$ & 0.53 & $1 \cdot 31^{\mathrm{ab}}$ & 0.62 & $1.92^{\mathrm{b}}$ & $0 \cdot 55$ \\
\hline Dietary Vitamin $B_{5}(\mu \mathrm{g} /$ day $)$ & $4 \cdot 66^{\mathrm{ab}}$ & 2.57 & $3 \cdot 35^{\mathrm{a}}$ & 1.58 & $4 \cdot 40^{\mathrm{ab}}$ & $1 \cdot 36$ & $6 \cdot 18^{\mathrm{b}}$ & 1.63 \\
\hline Age (years) & $39 \cdot 33^{\mathrm{ab}}$ & $11 \cdot 85$ & $48 \cdot 33^{\mathrm{b}}$ & $10 \cdot 24$ & $44 \cdot 77^{\mathrm{ab}}$ & 9.93 & $36 \cdot 50^{\mathrm{a}}$ & 5.69 \\
\hline Working hours/week & $49 \cdot 67^{\mathrm{c}}$ & $8 \cdot 39$ & $35 \cdot 57^{\mathrm{bc}}$ & 11.06 & $23.81^{\mathrm{a}}$ & 13.99 & $28 \cdot 06^{\mathrm{ab}}$ & 6.62 \\
\hline
\end{tabular}

* Individual mean was calculated based on the 3-day dietary record with all resulted values being classified into four levels. Different uppercase superscript indicate statistically significant differences amongst snacking-frequency groups.

In conclusion, total energy and certain macronutrient intakes followed an increasing trend with the increased snacking frequencies. Snacking of four times a day resulted in significantly higher intakes of vitamin $\mathrm{B}_{2}$ and $\mathrm{B}_{5}$ compared to the snacking of twice a day group but stayed the lowest in the total carotene intake. Snacking of the highest frequency was observed in the youngest age group who worked for almost the shortest working time per week amongst all.

1. Murakami K and Livingstone MB (2016) Br J Nutr March 22, 1-11.

2. Park E, Edirisinghe I, Inui T et al. Physio Behav 159, 64-71. 